Proceedings of the International School and Conference on Optics and Optical Materials, ISCOM07, Belgrade, Serbia, September 3-7, 2007

\title{
Surface Plasmon-Polariton Assisted Metal-Dielectric Multilayers as Passband Filters for Ultraviolet Range
}

\author{
Z. JAKŠI $\dot{c}^{a, *}$, M. MAKSimović ${ }^{b}$, M. SARAJlić ${ }^{a}$ \\ AND D. TANASKOVIĆ ${ }^{a}$ \\ ${ }^{a}$ Institute of Microelectronic Technologies and Single Crystals \\ Institute of Chemistry, Technology and Metallurgy \\ University of Belgrade, Serbia \\ ${ }^{b}$ Dept. of Applied Mathematics, University of Twente, The Netherlands \\ We designed and fabricated metal-dielectric multilayers intended for \\ passband filters in the ultraviolet range. We determined the dispersion char- \\ acteristics by the Bloch approach to evanescent wave resonant coupling and \\ calculated the spectral characteristics using the transfer matrix method while \\ taking into account real dispersion and absorptive losses. We considered the \\ influence of nanoscale interface roughness as a means to couple evanescent \\ electromagnetic field to the propagating far field modes. In our structures \\ both propagating and evanescent modes contribute to the overall perfor- \\ mance, resulting in an enhanced transmission in the desired range, while \\ retaining a strong suppression of undesired frequencies of more than four \\ orders of magnitude. In our experiments we used radiofrequent sputtering \\ of silver and silica and characterized our multilayers by UV-vis spectroscopy.
}

PACS numbers: 42.79.Wc, 42.79.Ci, 42.25.Bs, 42.25.Hz, 42.70.Qs

\section{Introduction}

Metal-dielectric multilayer thin films have been traditionally used for optical filtering [1]. A renewed interest for this field has been created with the advent of nanoplasmonics and with the introduction of left-handed metamaterials and metal-dielectric superlenses [2, 3] including those with metal-dielectric multilayers. Scalora et al. [4] experimentally showed that under certain condition metal-dielectric multilayers (which they termed transparent metals) transmit light by resonant tunneling, enabling extraordinary transmission. 1D metal-dielectric

*corresponding author; e-mail: jaksa@nanosys.ihtm.bg.ac.yu 
nanofilm multilayers for the UV range were analyzed theoretically and experimentally in [5].

In this work we analyzed a possibility to simultaneously utilize interference of propagating waves and plasmon resonance to improve performance of silver-silica multilayer filters for UV range. We determined the dispersion characteristics of our structures and chose a frequency range where propagating and plasmon modes overlap. We applied transfer matrix method to calculate the transmission due to interference of propagating waves. We fabricated our structures by RF sputtering.

\section{Theory}

The geometry of our metal-dielectric multilayers is shown in Fig. 1. It is a 1D analogy of metal-dielectric photonic crystal; this structure represents the standard configuration for multilayer superlenses $[3,6]$. However, a difference is that the interfaces between the layers here are roughened on subwavelength level. For the operating wavelengths in the ultraviolet and visible the roughness was about $30 \mathrm{~nm}$.

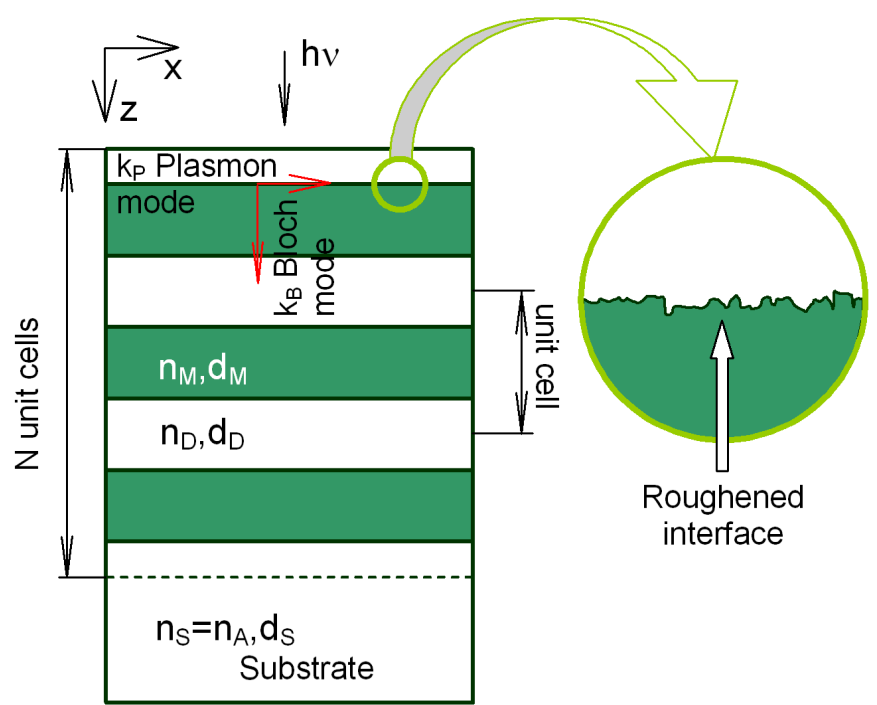

Fig. 1. Basic metallodielectric multilayer. Shaded - metal, white - dielectric. A single unit cell starts and ends with half-thickness dielectric. The inset displays a magnified interface to show its subwavelength roughening.

The unit cell of the 1D multilayer was symmetric, as shown in Fig. 1. This is due to the fact that a half-thickness dielectric layer was deposited on the top of the structure.

The geometry enables the propagation of the incident wave simultaneously in two ways. One of them is through the simple interference of propagating waves 
and is determined through the application of the transfer matrix method. Another is through the coupled plasmon waveguide resonance [7, 8]. For this to be effective, surface roughness must serve as a coupler between the input propagating wave and the in-plane plasmon wave. According to Raether [9], the surface roughness can be regarded as a superposition of a number of diffractive gratings with various parameters and can serve as a coupling means between the propagating and evanescent waves. The in-plane component of plasmons thus obtained at the input metal-dielectric interface resonantly couples with the waves on the further metal layers and the result is the Bloch propagation of the evanescent modes $[7,8]$. Finally, the surface roughness at the exit interface again converts electromagnetic energy from plasmons back to the output propagating mode. For this to be effective, a range of wave vector values in the dispersion relation must exist where both propagating and evanescent modes coexist.

The dispersion relation for the Bloch waves is obtained in the form [10]:

$$
\begin{aligned}
& \cos \left(k_{\mathrm{B}} d\right)=\cosh \left(\alpha_{\mathrm{SiO}_{2}} d_{\mathrm{SiO}_{2}}\right) \cosh \left(\alpha_{\mathrm{Ag}} d_{\mathrm{Ag}}\right) \\
& \quad+\frac{\alpha_{\mathrm{SiO}_{2}}^{2} \varepsilon_{\mathrm{Ag}}^{2}+\alpha_{\mathrm{Ag}}^{2} \varepsilon_{\mathrm{SiO}_{2}}^{2}}{2 \alpha_{\mathrm{SiO}_{2}} \alpha_{\mathrm{Ag}} \varepsilon_{\mathrm{SiO}_{2}} \varepsilon_{\mathrm{Ag}}} \sinh \left(\alpha_{\mathrm{SiO}_{2}} d_{\mathrm{SiO}_{2}}\right) \sinh \left(\alpha_{\mathrm{Ag}} d_{\mathrm{Ag}}\right), \\
& \alpha_{\mathrm{SiO}_{2}}^{2}=k_{x}^{2}-\left(\frac{\omega}{c}\right)^{2} \varepsilon_{\mathrm{SiO}_{2}} \mu_{\mathrm{SiO}_{2}}, \quad \alpha_{\mathrm{Ag}}^{2}=k_{x}^{2}-\left(\frac{\omega}{c}\right)^{2} \varepsilon_{\mathrm{Ag}} \mu_{\mathrm{Ag}} .
\end{aligned}
$$

The condition for the existence of propagating Bloch modes is $\left|\cos \left(k_{\mathrm{B}} d\right)\right| \leq 1$. The Bloch modes are propagating if $\alpha_{i}^{2}<0$ and are evanescent if $\alpha_{i}^{2}>0$.

\section{Calculation}

We performed our calculations for a silver-silica material pair. We utilized our fit [5] for the real and imaginary component of $\mathrm{Ag}$ dielectric permittivity. We determined the dispersion characteristics for our multilayer according to (1), (2) [10] (Fig. 2). We applied the transfer matrix method (TMM) taking into account

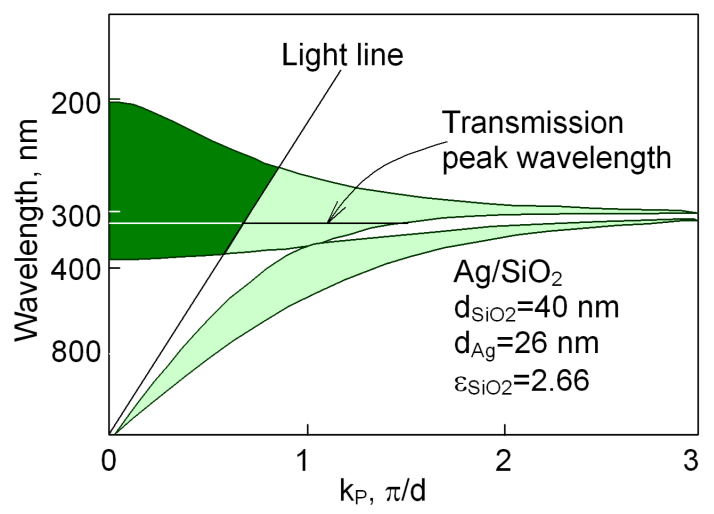

Fig. 2. Calculated spectral dispersion for the silver/silica pair. Dark - propagating modes; light — evanescent modes. 
dispersion and losses [11] to determine the spectral characteristics of the multilayer for the propagating modes.

\section{Experimental}

We used unpolished fused silica as the substrate. The idea was to utilize the natural roughness of the unpolished samples as the coupling means between the propagating modes and the surface plasmons in a manner equivalent to the use of diffractive grating coupler.

After rinsing and drying the silica plates we deposited alternating layers of silver $(26 \mathrm{~nm})$ and silicon dioxide $(40 \mathrm{~nm})$ on our Perkin Elmer RF sputtering system. We kept the argon pressure at $2 \times 10^{-5}$ bar and used the operating mode without substrate rotation and without substrate heating. Silicon dioxide was deposited for $4 \mathrm{~min}$ at a cathode voltage of $1200 \mathrm{~V}$ and at $1 \mathrm{~kW}$ RF power. Silver was deposited during $25 \mathrm{~s}$ at a cathode voltage of $1100 \mathrm{~V}$ and at $0.5 \mathrm{~kW}$ RF power. We fabricated samples with 1, 2, 3, and 4 layer pairs. At the top of each multilayer we deposited a silica layer with a half of the thickness used in the multilayer. Thus a symmetrical unit cell was obtained - i.e. each unit cell both starts and ends with a silica layer with a $1 / 2$ full thickness. This is a condition for the existence of the Bloch transmission of the resonant plasmons [11].

The reflection and transmission spectra of the obtained samples were measured on a UV-vis spectrometer Thermo-Nicolet (Fig. 3). The surface morphology was characterized by atomic force microscopy (Fig. 4). It can be seen that the roughness is preserved after sputtering 4 layer pairs.
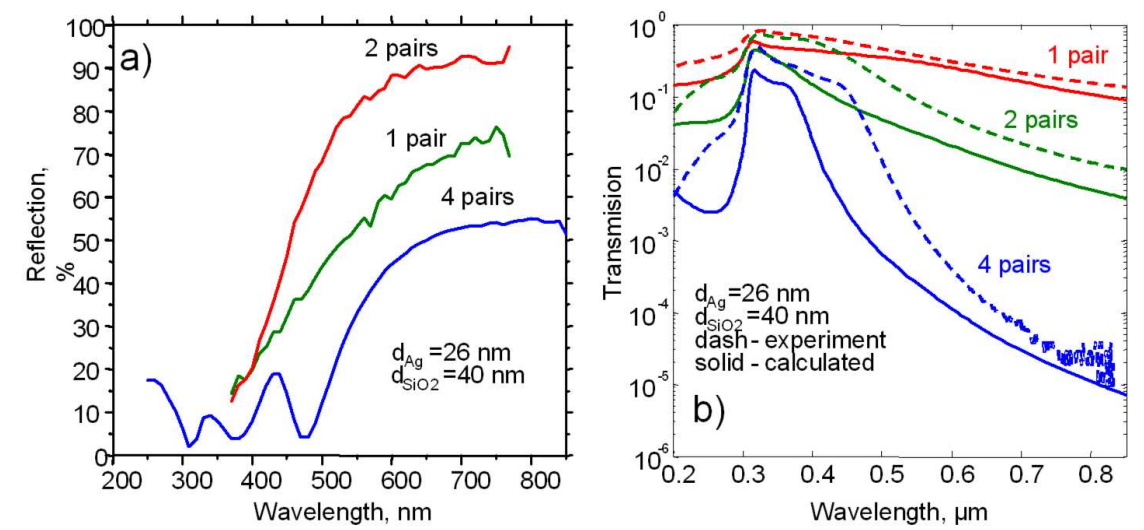

Fig. 3. (a) Measured reflection of silver-silica nanofilm multilayers for different numbers of layer pairs; (b) Calculated using TMM (solid) and measured (experimental) spectral transmission of silver-silica nanofilm multilayers for different numbers of layer pairs.

A comparison between the calculated and measured spectral transmissions (Fig. 3b) shows that the experimental values exceed somewhat the ideal theoretical values obtained without taking into account plasmon coupling at the metal- 



Fig. 4. AFM images of the sample surfaces: (a) fused silica substrate and (b) metaldielectric multilayer with 4 layer pairs.

dielectric interfaces. It is our opinion that this improvement can be contributed to the plasmon resonant coupling, as outlined in the previous text. The obtained filters have an excellent suppression of undesired frequencies and a high transmission at the desired peak. However, due to the stochastic nature of surface roughening, the resulting enhancement has to be a tradeoff between coupling through the equivalent diffractive gratings formed as harmonic of the total roughness and the diffusive scattering due to the same roughness. Thus one could envision further improvement of the filter performance if an ordered diffractive patterning of the interfaces were done, in a manner somewhat similar to the one encountered in subwavelength holes with extraordinary transmission.

\section{Conclusion}

We performed a theoretical and experimental analysis of metal-dielectric multilayer filters with roughened surfaces. Excellent filtering characteristics were obtained, with a suppression of undesired frequencies exceeding 4 orders of magnitude. Our current work is dedicated to the quantitative analysis of the contribution of the surface corrugation to the total spectral transmission of the multilayers.

Our further research, already partially underway, should include various generalizations of the multilayers. Since metals are materials with negative dielectric permittivity near the plasma frequency, an obvious direction is to consider replacing metal layers by material with both permittivity and permeability negative - i.e. by double negative (left-handed) metamaterial. Another is the use of nanostructured plasmonic surfaces instead of rough metal interface in order to controllably obtain designer plasmons with freely tailorable dispersion. 


\section{References}

[1] H.A. Macleod, Thin-Film Optical Filters, Institute of Physics Publishing, Bristol 2001.

[2] J.B. Pendry, Phys. Rev. Lett. 85, 3966 (2000).

[3] S.A. Ramakrishna, Rep. Prog. Phys. 68, 449 (2005).

[4] M. Scalora, M.J. Bloemer, A.S. Pethel, J.P. Dowling, C.M. Bowden, A.S. Manka, J. Appl. Phys. 83, 2377 (1998).

[5] Z. Jakšić, M. Maksimović, M. Sarajlić, J. Opt. A, Pure Appl. Opt. 7, 51 (2005).

[6] N. Fang, H. Lee, C. Sun, X. Zhang, Science 308, 534 (2005).

[7] S. Feng, J.M. Elson, P.L. Overfelt, Phys. Rev. B 72, 085117 (2005).

[8] A. Yariv, Y. Xu, R.K. Lee, A. Scherer, Opt. Lett. 24, 711 (1999).

[9] H. Raether, Surface Plasmons on Smooth and Rough Surfaces and on Gratings, Springer-Verlag, Berlin 1986.

[10] S. Feng, J.M. Elson, P.L. Overfelt, Opt. Express 13, 4113 (2005).

[11] M. Maksimović, Z. Jakšić, Phys. Lett. A 342, 497 (2005). 